Pacific Journal of Mathematics

INVARIANT MEANS ON TOPOLOGICAL SEMIGROUPS 


\section{INVARIANT MEANS ON TOPOLOGICAL SEMIGROUPS}

\section{Argabright}

This paper is concerned with the existence and structure of invariant means on the space $C(S)$ of all (including unbounded) continuous real-valued functions on a topological semigroup $S$. The main result is that for realcompact semigroups every left invariant mean (if any exist) arises as an integral over a compact left invariant subset of $S$. The question of existence of noncompact group $G$ such that $C(G)$ admits a left invariant mean is also considered. If $G$ is a realcompact (or discrete, or locally compact abelian) group, then $C(G)$ admits a left invariant mean only if $G$ is compact.

By a topological semigroup we mean a semigroup $S$ endowed with a Hausdorff topology for which the mapping $(x, y) \rightarrow x y$ of $S \times S$ into $S$ is continuous. We denote by $C(S)$ the space of all continuous real-valued functions on $S$ (not necessarily bounded). If $a \in S$ and $f \in C(S)$, then ${ }_{a} f$ and $f_{a}$ will denote those functions on $S$ whose values at $x \in S$ are $f(a x)$ and $f(x a)$ respectively. Obviously ${ }_{a} f$ and $f_{a}$ are elements of $C(S)$. A left invariant mean on $C(S)$ is a nonnegative linear functional $M$ on $C(S)$ such that that $M(1)=1$ and $M\left({ }_{a} f\right)=M(f)$ for all $a \in S$ and $f \in C(S)$. Right invariant means are defined similarly, replacing ${ }_{a} f$ by $f_{a}$. A functional $M$ that is both a left invariant mean and a right invariant mean is called a two-sided invariant mean.

The purpose of this to study the structure of invariant means on $C(S)$. This problem differs from the usual problem of finding invariant means for semigroups (see reference [2]), which concerns invariant means on the space $C^{*}(S)$ of all bounded continuous real-valued functions on $S$. Of course $S$ is pseudocompact, then $C(S)=C^{*}(S)$ and the two problems are the same. Our main result is to the effect that, for a large class of semigroups $S$, every left invariant mean (if any exist) arises in a particularly simple way; namely, as an integral over a compact left invariant subset of $S$. In the final section we consider the question of whether there exist noncompact groups $G$ such that $C(G)$ admits a left invariant mean. A negative answer is obtained for noncompact groups of various types.

In this section we establish some terminology and preliminary facts needed for the statement and proof of the main theorem.

Received May 17, 1964. This paper is based on a portion of the author's doctoral thesis prepared under the direction of Professor Edwin Hewitt at the University of Washington. The author wishes to express his appreciation to Professor Hewitt and Professor J. Isbell for their interest and helpful suggestions. 
THEOREM 1.1. Let $F$ be a compact right simple semigroup. ${ }^{1}$ Then $F$ is topologically isomorphic to a direct product $G \times E$ where $G$ is a compact topological group and $E$ is a compact right zero semigroup. ${ }^{1}$

Proof. Numakura ([10], p. 103) has proved that a compact semigroup contains at least one idempotent. Let $E$ be the set of all idempotents in $F$ and let $G=F e_{0}$, where $e_{0}$ is a fixed member of $E$. It is easy to see that $E$ and $G$ are compact and that $e x=x$ for all $e \in E, x \in F$. Moreover, (see [1], Theorem 1.27, p. 38) $G$ is a group and $G \times E$ is isomorphic to $F$ via the mapping $\left(x e_{0}, e\right) \rightarrow(x e) e=x e$. This mapping is obviously continuous and hence is also a homeomorphism. Finally, since $G$ is compact and multiplication in $G$ is jointly continuous, it follows that inversion is also continuous in $G$. Hence $G$ is a topological group.

The following theorem is due (independently) to $E$. Hewitt and W. G. Rosen. The statement and proof given here are taken from Hewitt's manuscript [8]. ${ }^{2}$ It can also be inferred from [12].

THEOREM 1.2. Let $F$ be a compact right simple semigroup and write $F=G \times E$ as in 1.1. Let $\sigma=\lambda \times \mu$ where $\lambda$ is the normalized Haar measure on $G$ and $\mu$ is any probability measure on $E .^{3}$ Then the mapping

$$
f \rightarrow \int_{F} f d \sigma
$$

is a left invariant mean on $C(F)$. Moreover, every left invariant mean on $C(F)$ is of this type.

Proof. For $f \in C(F)$, let $M(f)=\int_{F} f d \sigma$. Obviously $M$ is nonnegative and linear. Moreover, by Fubini's Theorem, we may write $M$ as an iterated integral

$$
M(f)=\int_{E}\left[\int_{\theta} f(x, e) d \lambda(x)\right] d \mu(e) .
$$

Note that for $(a, d) \in G \times E$ we have

$$
{ }_{(a, a)} f(x, e)=f(a x, d e)=f(a x, e) .
$$

${ }^{1}$ We follow the terminology of Clifford and Preston [1]. A semigroup $F$ is right simple if $a F=F$ for all $a \in F$; a semigroup $E$ is called a right zero semigroup if $x y=y$ for all $x, y \in E$.

2 The author wishes to especially thank Professor Hewitt for allowing him to read his unpublished manuscript.

3 A regular Borel measure such that $\mu(E)=1$. 
Thus by invariance of the Haar integral on $G$ we have

$$
\begin{aligned}
M\left(_{(a, a)} f\right) & =\int_{E}\left[\int_{G} f(a x, e) d \lambda(x)\right] d \mu(e) \\
& =\int_{E}\left[\int_{G} f(x, e) d \lambda(x)\right] d \mu(e)=M(f) .
\end{aligned}
$$

Hence $M$ is a left invariant mean on $C(F)$.

Conversely, suppose $M$ is a left invariant mean on $C(F)$. For arbitrary functions $\phi \in C(G)$ and $\psi \in C(E)$, let $\Phi$ and $\Psi$ be the functions on $F=G \times E$ defined by $\Phi(x, e)=\varphi(x)$ and $\Psi(e, x)=\Psi(e)$ respectively. Obviously $C_{1}=\{\Phi: \varphi \in C(G)\}$ and $C_{2}=\{\Psi: \psi \in C(E)\}$ are linear subspaces of $C(F)$ which are isometrically isomorphic to $C(G)$ and $C(E)$ respectively. Hence, by the Riesz representation theorem, there exist probability measures $\lambda$ and $\mu$ on $G$ and $E$ respectively such that

$$
\begin{aligned}
& M(\Phi)=\int_{G} \varphi(x) d \lambda(x) \text { for all } \varphi \in C(G) \\
& M(\Psi)=\int_{E} \psi(x) d \mu(x) \text { for all } \psi \in C(E) .
\end{aligned}
$$

Moreover, since ${ }_{(a, d)} \Phi(x, e)=\Phi(a x, e)=\varphi(a x)$ and $M$ is left invariant, we have $\int_{G} \varphi(a x) d \lambda(x)=\int_{G} \varphi(x) d \lambda(x)$ for all $\varphi \in C(G, R)$ and $a \in G$. Thus $\lambda$ is, in fact, Haar measure on $G$ and $H(\varphi)=\int \varphi(x) d \lambda(x)$ is the Haar integral on $G$.

Now let us consider functions of the form $\Phi \cdot \Psi$. Note that

$$
\begin{aligned}
{ }_{(a, a)}(\Phi \cdot \Psi)(x, e) & =\Phi(a x, e) \cdot \Psi(a x, e)=\varphi(a x) \cdot \psi(e) \\
& \left.={ }_{(a, d)} \Phi\right) \cdot \Psi(x, e)
\end{aligned}
$$

and so

$$
M(\Phi \Psi)=M\left(\left(_{(a, d)} \Phi\right) \cdot \Psi\right) .
$$

More generally, for any finite sequence $a_{1}, \cdots, a_{n}$ of elements of $G$, we have

$$
M(\Phi \Psi)=M\left(\left(\frac{1}{n} \sum_{i=1}^{n}\left(a_{i}, d\right) \Phi\right) \cdot \Psi\right)
$$

By a well-known construction of the Haar integral on a compact group (see [11]), there is a sequence of functions of the form $1 / n \sum_{i=1 a_{i}}^{n} \rho$ which converge uniformly to the constant function $H(\varphi)$ on $G$. The corresponding functions $1 / n \sum_{i=1\left(a_{i}, a\right)}^{n} \Phi$ converge uniformly to the constant function $H(\varphi)$ on $F$. Thus, since $M$ is continuous in the uniform topology on $C(F)$, we have 


$$
\begin{aligned}
M(\Phi \Psi) & =H(\varphi) M(\Psi)=\left(\int_{G} \varphi(x) d(\lambda(x))\right) \cdot\left(\int_{E} \psi(e) d \mu(e)\right) \\
& =\int_{E}\left[\int_{G} \Phi \Psi(x, e) d \lambda(x)\right] d \mu(e) \\
& =\int_{F} \Phi \Psi d \sigma
\end{aligned}
$$

where $\sigma$ denotes the product measure $\lambda \times \mu$. Furthermore, for any function of the from $\sum_{i=1}^{n} \Phi_{i} \Psi_{i}$, we have

$$
\begin{aligned}
M\left(\sum_{i=1}^{n} \Phi_{i} \Psi_{i}\right) & =\sum_{i=1}^{n} M\left(\Phi_{i} \Psi_{i}\right)=\sum_{i=1}^{n} \int_{F} \Phi_{i} \Psi_{i} d \sigma \\
& =\int_{F} \sum_{i=1}^{n} \Phi_{i} \Psi_{i} d \sigma
\end{aligned}
$$

and, since the latter class of functions is uniformly dense in $C(F)$, we conclude that the equality

$$
M(f)=\int_{F} f d \sigma
$$

holds for all $f \in C(F)$. This completes the proof.

1.3. THEOREM. Let $S$ be an arbitrary topological semigroup and suppose $S$ contains a compact left invariant subset $F .^{4}$ Let $\sigma$ be a product measure on $F$ as in 1.2. Then the mapping $f \rightarrow \int_{F} f d \sigma$ is a left invariant mean on $C(S)$.

Proof. Let $N(\varphi)=\int_{F} \varphi d \sigma$ for all $\varphi \in C(F)$, and let $M(f)=$ $N\left(\left.f\right|_{F}\right)$ for all $f \in C(S)$. Obviously $M$ is nonnegative and linear, and $M(1)=1$. We have only to show that $M$ is left invariant. Since $F^{\prime}$ is a compact subsemigroup of $S$, it contains at least one idempotent $e$. Moreover ([1], p. 37), $e$ is a left identity in $F$. Thus, for each $a \in S$ and $f \in C(S)$, we have

$$
{ }_{a} f(x)=f(a x)=f(a(e x))=f((a e) x)={ }_{a e} f(x)
$$

for all $x \in F$; that is, $\left.{ }_{a} f\right|_{F}={ }_{a e}\left(\left.f\right|_{F}\right)$. Since $a e \in F$ and $N$ is left invariant on $C(F)$, if follows that

$$
M\left(_{a} f\right)=N\left(\left.{ }_{a} f\right|_{F}\right)=N\left({ }_{a e}\left(\left.f\right|_{F}\right)\right)=N\left(\left.f\right|_{F}\right)=M(f) .
$$

2. The main theorem. In this section we show that if $S$ is a realcompact semigroup then every left invariant mean on $C(S)$ (if any exist) arises as in 1.3. For the definition of "realcompact" see [3] or

${ }^{4}$ By this we mean that $a F=F$ for all $a \in S$; equivalently, $F$ is a right simple left ideal in $S$. 
[6]..$^{5} \quad$ We remark (see [3]) that any completely regular Lindelof space is realcompact and that products and closed subspaces of realcompact spaces are realcompact; also any discrete space of nonmeasurable cardinal is realcompact.

The proof of our main theorem is based on a theorem of Hewitt [7] concerning the representation of certain linear functionals on spaces of continuous functions. ${ }^{6}$ We begin by summarizing Hewitt's result in the form in which we shall apply it.

\subsection{An integral representation of linear functionals (see [7]).}

Let $X$ be a completely regular space and let $C(X)$ denote the set of all continuous real-valued functions on $X$. For $f \in C(X)$, let $P(f)=$ $\{x \in X: f(x)>0\}$ and let $Z(f)=\{x \in X: f(x)=0\}$. Let $\mathscr{P}(X)$ denote the family of all sets $P(f)$ and $\mathscr{Z}(X)$ the family of all sets $Z(f)$. The smallest $\sigma$-algebra of subsets of $X$ containing $\mathscr{P}(X)$ is called the family of Baire sets of $X$. A Baire measure on $X$ is a countably additive set function defined on Baire sets. If $I$ is a nonnegative linear functional on $C(S)$ such that $I(1)=1$, then there exists a Baire measure $\gamma$ on $X$ such that $\gamma(X)=1$ and

$$
I(f)=\int_{x} f(x) d \gamma x
$$

for all $f \in C(X)$. The measure $\gamma$ has the property that every function $f \in C(X)$ is bounded except on a set (depending on the function) of $\gamma$ measure zero. Moreover, if $X$ is realcompact, then the set

$$
F=\cap\{Z \in \mathscr{Z}(X): \gamma(Z)=1\}
$$

is nonvoid and compact. This compact set $F$ supports $\gamma$ (or $I$ ) in the sense that if $G \in \mathscr{P}(X)$, then $\gamma(G)>0$ if and only if $G \cap F \neq \varnothing$. It follows from this that if $f, g \in C(X)$ and $f(x)=g(x)$ for all $x \in F$, then $I(f)=I(g)$.

We now state and prove our result.

THeOREM 2.2. Let $S$ be a realcompact semigroup. Then every left invariant mean on $C(S)$ (if any exist) is of the form 1.3.

Proof. Suppose $M$ is a left invariant mean on $C(S)$. Then there exists a Baire measure $\gamma$ on $X$ with compact support $F$ (in the sense described in 2.1) such that $M(f)=\int_{S} f d \gamma$ for all $f \in C(S)$. We will show that $F$ is a left invariant subset of $S$ and that $M$ can be represented as an integral over $F$ as in 1.3.

5 "Realcompact space" means the same as " $Q$-space" in Hewitt's terminology.

${ }^{6}$ Also see [5]. 
We first show that $a F \subset F$ for all $a \in S$. Let $Z$ be an arbitrary element of $\mathcal{Z}(X)$ such that $\gamma(Z)=1$, and write $Z=Z(f), f \geqq 0$. Then

$$
M(f)=\int_{z} f d \gamma+\int_{Z^{\prime}} f d \gamma=0+0=0,
$$

and so

$$
M\left({ }_{a} f\right)=\int_{X} f(a x) d \gamma(x)=0 .
$$

Since $f$ is continuous and $\geqq 0$, it follows that $f(a x)=0$ for all $x \in F$. Thus $a F \subset Z(f)=Z$ and, since $Z$ was arbitrary, we have

$$
a F \subset \cap\{Z \in \mathscr{Z}(X): \gamma(Z)=1\}=F .
$$

We now show that $F$ is left invariant; that is, $a F=F$ for all $a \in S$. Suppose not. Then there exists $a \in S$ and $b \in F$ such that $a x \neq b$ for all $x \in F$. Thus, for each $x \in F$, there is a continuous function $f: S \rightarrow[0,1]$ such that $f(b)=1$ and $f(a x)=0$. Let

$$
g=2\left[\max \left(f, \frac{1}{2}\right)-\frac{1}{2}\right]
$$

and note that $g(b)=1$ and $g(a y)=0$ for all

$$
y \in U(x)=\left\{y \in S: f(a y)<\frac{1}{2}\right\}=P\left(\frac{1}{2}-{ }_{a} f\right) .
$$

Since $F$ is compact, there exist points $x_{1}, \cdots, x_{n} \in F$ such that $F \subset U\left(x_{1}\right) \cup \cdots \cup U\left(x_{n}\right)$. Let $g_{1}, \cdots, g_{n}$ be the corresponding functions $g$ and let

$$
h=\min \left(g_{1}, \cdots, g_{n}\right) .
$$

Then $h \in C(S), h: S \rightarrow[0,1], h(b)=1$, and $h(a x)=0$ for all $x$ it the set $U=U\left(x_{1}\right) \cup \cdots \cup U\left(x_{n}\right)$. We note that $U \in \mathscr{P}(X)$ and hence is $\gamma$ measurable. It follows that

$$
M(h)=\int_{S} h(x) d \gamma(x)>0
$$

whereas

$$
M\left({ }_{a} h\right)=\int_{U} h(a x) d \gamma(x)+\int_{U^{\prime}} h(a x) d \gamma(x)=0+0=0,
$$

a contradiction.

It remains only to show that there is a product measure $\sigma$ on $F$ such that $M$ is of the form 1.3. We note that $F$ (being compact) is $C$-embedded in $S([3]$, p. 43$)$; that is, every function in $C(F)$ can be 
extended to a function in $C(S)$. Also, for any $f \in C(S), M(f)$ is completely determined the values of $f$ on $F$, that is, if $g=f$ on $F$, then $M(g)=M(f)$. Thus $M$ induces a linear functional $L$ on $C(F)$ as follows: If $\varphi \in C(F)$, let $L(\varphi)=M(f)$ where $f$ is any function in $C(S)$ such that $\left.f\right|_{F}=\varphi$. It is easy to check that $L$ is a left invariant mean on $C(F)$. Hence, by 1.2, $L$ has the form $L(\varphi)=\int_{F} \phi d \sigma$ where $\sigma$ is a product measure on $F$ as in 1.2. Finally, we have

$$
M(f)=L\left(\left.f\right|_{F}\right)=\int_{F} f d \sigma
$$

for all $f \in C(S)$. This completes the proof.

Obviously, results completely analogous to $1.2,1.3$, and 2.2 hold for right invariant means. For two-sided invariant means we have the following.

CoRollary 2.3. Let $S$ be a topological semigroup and suppose $S$ contains a compact subgroup $G$ which is a two-sided ideal in $S$. Let $\gamma$ denote the normalized Haar measure on $G$. Then

$$
f \rightarrow \int_{G} f d \gamma
$$

is a two-sided invariant mean on $C(S)$. Moreover, if $S$ is realcompact, then every two-sided invariant mean on $C(S)$ (if any exist) is of this type.

Obviously, left invariant means on $C(S)$ (when they exist) are not in general unique. However, we do have the following.

THEOREM 2.4. Let $S$ be a realcompact semigroup. If $C(S)$ admits a left invariant mean $M$ and a right invariant mean $L$, then $M=L$. In particular, two-sided means (when they exist) are unique.

Proof. By 2.2, $S$ contains a compact left invariant subset $F_{1}$ such that $M(f)=\int_{F_{1}} f d \sigma$ as in 1.3. Analogously, $S$ contains a compact right invariant set $F_{2}$ such that $L(f)=\int_{F_{2}} f d \tau$ where $\tau$ is a product measure on $F_{2}$ analogous to the measure $\sigma$. But, obviously, $F_{2}=F_{2} F_{1}=F_{1}$. Thus

$$
M(f)=L(f)=\int_{\theta} f d \lambda
$$

where $G=F_{1}=F_{2}$ is a compact group and $\lambda$ is the normalized Haar measure on $G$. 
EXAMPLE 2.5. A nonrealcompact semigroup $S$ may admit a left invariant mean which cannot be represented as in 1.3. For example, let $\Omega$ denote the first uncountable ordinal and let $S$ be the set of all ordinals $\alpha<\Omega$, endowed with the order topology and a "multiplication" defined by $\alpha \beta=\max (\alpha, \beta)$. It is easy to see that $S$ is a topological semigroup and that $M(f)=\lim _{\alpha \rightarrow \Omega} f(\alpha)^{7}$ is a two-sided invariant mean on $C(S)$. However, $M$ cannot be represented as in 1.3 since $S$ contains no compact invariant subset.

It $S$ is an arbitrary completely regular semigroup and $M$ is a left invariant mean on $C(S)$, then we can also regard $M$ as a linear functional on $C(u S){ }^{8}$ As such, it has an integral representation

$$
M(f)=\int_{v S} f^{v}(x) d \gamma(x)
$$

where $\gamma$ is a Baire measure on $u S$ having compact support $F$ (in $v S$ ) which is "left invariant" in the sense that $L_{a}^{v}(F)=F$ for all $a \in S$; here $L_{a}$ denotes the mapping $x \rightarrow a x$ of $S$ into $S$. One can obtain a proof of this fact by modifying the proof of 2.2 in an obvious way. We note that if $S$ and $M$ are as in 2.5 , then $\cup S$ can be realized as the space of all ordinals less than or equal to $\Omega$ (with the order topology) and we have $M(f)=f^{v}(\Omega)$ for all $f \in C(S)$.

Finally, we use 2.2 to derive the following theorem on invariant means over compact semigroups. This result was obtained originally by Hewitt [8] and Rosen [12]. Recall (see [10], p. 104) that a compact semigroup $S$ contains a unique minimal ideal $K$, called the kernel of $S$, which is compact.

THEOREM 2.6. Let $S$ be a compact semigroup. Then $C(S)$ admits a left invariant mean if and only if $K$ (the kernel of $S$ ) is right simple. All left invariant means (if any exist) are of the form 1.3 where $F \subset K$.

Proof. If $K$ is right simple, then $C(S)$ admits a left invariant mean by 1.3. Conversely, suppose $C(S)$ admits a left invariant mean $M$. Then, by 2.2 , there is a compact left invariant subset $F$ in $S$ such that $M$ is of the form 1.3. Since $F$ is left invariant and $K$ is a right ideal we have $K F \subset K \cap F$; hence $K \cap F \neq \varnothing$. Let a $\in K \cap F$. Then $F=a F \subset K$. It remains to show that $K$ is right simple. We note that $F K$ is a two-sided ideal contained in $K$; hence $F K=K$.

7 This limit always exists; in fact, every function in $C(S)$ is eventually constant ([3], p. 75).

8 The space $u S$ is a realcompact space in which $S$ is dense and which has the property that every continous mapping $\tau$ from $S$ into any realcompact space $Y$ has a continuous extension $\tau^{v}$ mapping $u S$ into $Y$ (see [3], p. 118). 
Then for each $x \in S$ we have

$$
x K=x(F K)=(x F) K=F K=K .
$$

Hence $K$ is right simple.

Corollary 2.7. Let $S$ be a compact semigroup. Then $C(S)$ admits a two-sided invariant mean if and only if $K$ (the kernel of $S)$ is a group. In this case the unique invariant mean is just the Haar integral over $K$.

3. Invariant means on groups. Harmonic analysis on compact groups $G$ depends heavily on the existence of a unique, positive, invariant mean on $C(G)$. Once conceivable extension to noncompact groups $G$ and their unbounded continuous representations would be via an invariant mean on $C(G)$. In this section we consider the question of whether there exist noncompact groups $G$ such that $C(G)$ admits a left invariant mean. We obtain a negative answer for noncompact groups of various types; namely, realcompact groups, discrete groups, and locally compact abelian groups.

EXAMPLE 3.1. We note that there do exist noncompact groups $G$ such that $C(G)$ admits a left invariant mean. In fact, since every commutative group $G$ admits an invariant mean on $C^{*}(G)$ (see [2], p. 516), it suffices to produce a noncompact commutative group which is pseudocompact. ${ }^{9}$ The existence of such groups was pointed out by Glicksberg in [4]. Specifically, let $P=\prod_{\lambda \in \Lambda} Z_{2}$ where $Z_{2}=\{0,1\}$ is the two element group and $\Lambda$ is an uncountable index set. Let $G$ the subgroup of $P$ consisting of all points $x \in P$ for which $x_{\lambda}=0$ for all but countably many $\lambda \in \Lambda$. Then $G$ is a nonclosed (hence noncompact) subgroup of $P$. On the other hand, $G$ is sequentially compact and hence pseudocompact.

It is also interesting to note that, for an arbitrary topological group $G, C(G)$ admits a left invariant mean if and only if it admits a right invariant mean: For each $f \in C(G)$ and $x \in G$, let $f^{*}(x)=f\left(x^{-1}\right)$. Then if $M$ is a left [right] invariant mean on $C(G)$, the functional $f \rightarrow M\left(f^{*}\right)$ is a right [left] invariant mean on $C(G)$.

THEOREM 3.2. Let $G$ be a realcompact group. Then $C(G)$ admits a left invariant mean only if $G$ is compact.

Proof. Suppose $C(G)$ admits a left invariant mean. Then, by

9 This author knows of no nonpseudocompact group $G$ for which $C(G)$ admits a left invariant mean. 
2.2, $G$. must contain a compact left invariant subset. But $G$, being a group, has no proper invariant subsets. Hence $G$ is compact.

We note that, barring measurable cardinals, ${ }^{10}$ every locally compact group is realcompact. Precisely: Every locally compact group $G$ is paracompact and hence admits a complete uniform structure ([9], p. 172 and p. 208). Thus, by Shirota's Theorem ([3], p. 229), $G$ is realcompact unless it contains a closed discrete subspace having measurable cardinal.

In view of this, 3.2 implies that, barring measurable cardinals, a locally compact group $G$ admits a left invariant on $C(G)$ only if $G$ is compact. The remainder of this section is devoted to showing that the restriction on cardinals can be avoided in the case of discrete groups or locally compact abelian group. We begin by observing that if $G$ is a completely regular topological group and if $M$ is a left invariant mean on $C(G)$, then the Baire measure $\gamma$ which represents $M$ (as in 2.1) is a left invariant measure. This can be verified by a routine computation using the definition of $\gamma$ in [7]. We omit the details.

Theorem 3.3. Let $G$ be an infinite discrete group. Then $G$ contains a subset $E$ such that $G$ is the union of a countably infinite family of pairwise disjoint left translates of $E$. Hence and

(i) G admits no nonzero, finite valued, left invariant measure,

(ii) $C(G)$ admits no left invariant mean.

Proof. Let $S$ be a countably infinite subset of $G$ and let $H$ be the subgroup generated by $S$. Then $H$ is countably infinite. Let the set $E$ be formed by selecting exactly one element from each right coset of $H$. Then $\{a E: a \in H\}$ is a countably infinite family of pairwise disjoint sets whose union is $G$.

Suppose now that $\mu$ is a left invariant measure on $G$. If $\mu(E)=0$, then $\mu(G)=\sum_{a \in H} \mu(a E)=\sum_{a \in H} \mu(E)=0$. If $\mu(E)>0$, then $\mu(G)=$ $\sum_{a \in B} \mu(E)=+\infty$. Hence $G$ admits no nonzero, finite valued, left invariant measure.

Our final theorem is based on the following:

Lemma 3.4. Suppose $G$ is a locally compact group which is not realcompact. Then $G$ contains an open and closed subgroup $H$ such that the left coset space $G / H$ has measurable cardinal.

10 The hypothesis that every cardinal is nonmeasurable is consistent with the usual axioms of set theory. 
Proof. Let $U$ be a neighborhood of $e$ (the identity in $G$ ) such that $U^{-1}=U$ and $U^{-1}$ is compact. Then $H=\bigcup_{n-1}^{\infty} U^{n}$ is an open and closed compactly generated subgroup of $G$. Note that $H$ is $\sigma$-compact and hence realcompact. If $G$ is not realcompact, then it contains a closed discrete subspace $D$ such that $\overline{\bar{D}}$ is measurable. Let $\left\{H_{\lambda}: \lambda \in \Lambda\right\}$ denote the family of all distinct left cosets of $H$. Then $\overline{\overline{D \cap \overline{H_{\lambda}}}}$ is nonmeasurable for all $\lambda$ (since $H_{\lambda}$ is realcompact).

On the other hand, $\overline{\bar{D}}=\sum_{\lambda \in \Lambda} \overline{\overline{D \cap H_{\lambda}}}$ and so $\overline{\bar{\Lambda}}$ must be measurable ([3], p. 164)

TheOREM 3.5. Let $G$ be a locally compact abelian group. Then $C(G)$ admits a left invariant mean only if $G$ is compact.

Proof. If $G$ is realcompact, then 3.2 applies. Otherwise, $G$ contains an open and closed subgroup $H$ such that the quotient group $G / H$ has measurable cardinal; in particular, $G / H$ is infinite. Suppose now that $M$ is a left invariant mean on $C(G)$. If, for each $f \in C(G / H)$ and $x \in G$, we let $f^{\prime}(x)=f(x H)$, then it is easy to see that $f \rightarrow M\left(f^{\prime}\right)$ is a left invariant mean on $C(G / H)$. This contradicts 3.3 (ii).

Hence $C(G)$ admits no left invariant mean.

\section{REFERENCES}

1. A. H. Clifford and G. B. Preston, The algebraic theory of semigroups, Amer. Math. Soc. Mathematical Surveys 7, Providence, R. I., 1961.

2. M. M. Day, Amenable semigroups, Illinois J. Math. 1 (1957), 509-544.

3. L. Gillman and M. Jerison, Rings of Continuous Functions, Van Nostrand, New York, 1960.

4. I. Glicksberg, Stone-Cech compactifications of products, Trans. Amer. Math. Soc. 90 (1959), 369-382.

5. G. G. Gould and M. Mahowald, Measures on completely regular spaces, Journal of the London Math. Soc. 37 (1962), 103-111.

6. E. Hewitt, Rings of real-valued continuous functions, Trans. Amer. Math. Soc. 64 (1948), 54-99.

7. - Linear functionals on spaces of continuous functions, Fund. Math. 37 (1950), 161-189.

8. - Invariant means of compact semigroups, unpublished manuscript.

9. J. L. Kelley, General Topology, Van Nostrand, New York, (1955).

10. K. Numakura, On bicompact semigroups, Math. J. Okayama University 1 (1952), 99-108.

11. L. Pontrjagin, Topological Groups, Princeton, 1939.

12. W. G. Rosen, On invariant means over compact semigroups, Proc. Amer. Math. Soc. 7 (1956) 1076-1082.

UNIVERSITY OF WASHINGTON

UNIVERSITY OF CALIFORNIA, BERKELEY 



\section{PACIFIC JOURNAL OF MATHEMATICS}

\section{EDITORS}

\section{H. SAMELSON}

Stanford University

Stanford, California

\section{R. M. BLUMenthaL \\ University of Washington \\ Seattle, Washington 98105}

*J. DugundjI

University of Southern California Los Angeles, California 90007

\section{RICHARD ARENS}

University of California

Los Angeles, California 90024

\section{ASSOCIATE EDITORS}
E. F. BECKENBACH
B. H. NeUManN
F. WOLF
K. YosIDA

\section{SUPPORTING INSTITUTIONS}

\author{
UNIVERSITY OF BRITISH COLUMBIA \\ CALIFORNIA INSTITUTE OF TECHNOLOGY \\ UNIVERSITY OF CALIFORNIA \\ MONTANA STATE UNIVERSITY \\ UNIVERSITY OF NEVADA \\ NEW MEXICO STATE UNIVERSITY \\ OREGON STATE UNIVERSITY \\ UNIVERSITY OF OREGON \\ OSAKA UNIVERSITY \\ UNIVERSITY OF SOUTHERN CALIFORNIA
}

\author{
STANFORD UNIVERSITY \\ UNIVERSITY OF TOKYO \\ UNIVERSITY OF UTAH \\ WASHINGTON STATE UNIVERSITY \\ UNIVERSITY OF WASHINGTON \\ AMERICAN MATHEMATICAL SOCIETY \\ CHEVRON RESEARCH CORPORATION \\ TRW SYSTEMS \\ NAVAL ORDNANCE TEST STATION
}

Mathematical papers intended for publication in the Pacific Journal of Mathematics should be typewritten (double spaced). The first paragraph or two must be capable of being used separately as a synopsis of the entire paper. It should not contain references to the bibliography. Manuscripts may be sent to any one of the four editors. All other communications to the editors should be addressed to the managing editor, Richard Arens at the University of California, Los Angeles, California 90024.

50 reprints per author of each article are furnished free of charge; additional copies may be obtained at cost in multiples of 50 .

The Pacific Journal of Mathematics is published monthly. Effective with Volume 16 the price per volume (3 numbers) is $\$ 8.00$; single issues, $\$ 3.00$. Special price for current issues to individual faculty members of supporting institutions and to individual members of the American Mathematical Society: $\$ 4.00$ per volume; single issues $\$ 1.50$. Back numbers are available.

Subscriptions, orders for back numbers, and changes of address should be sent to Pacific Journal of Mathematics, 103 Highland Boulevard, Berkeley 8, California.

Printed at Kokusai Bunken Insatsusha (International Academic Printing Co., Ltd.), No. 6, 2-chome, Fujimi-cho, Chiyoda-ku, Tokyo, Japan.

PUBLISHED BY PACIFIC JOURNAL OF MATHEMATICS, A NON-PROFIT CORPORATION

The Supporting Institutions listed above contribute to the cost of publication of this Journal, but they are not owners or publishers and have no responsibility for its content or policies.

* Paul A. White, Acting Editor until J. Dugundji returns. 


\section{Pacific Journal of Mathematics}

\section{Vol. 16, No. 2 December, 1966}

Loren N. Argabright, Invariant means on topological semigroups ........ 193

William Arveson, A theorem on the action of abelian unitary groups ...... 205

John Spurgeon Bradley, Adjoint quasi-differential operators of Euler

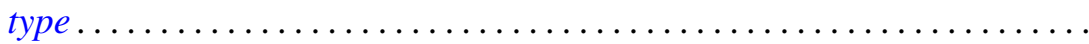

Don Deckard and Lincoln Kearney Durst, Unique factorization in power series rings and semigroups ........................... 239

Allen Devinatz, The deficiency index of ordinary self-adjoint differential operators..................................... 243

Robert E. Edwards, Operators commuting with translations ............ 259

Avner Friedman, Differentiability of solutions of ordinary differential equations in Hilbert space .............................. 267

Boris Garfinkel and Gregory Thomas McAllister, Jr., Singularities in a variational problem with an inequality ......................

Seymour Ginsburg and Edwin Spanier, Semigroups, Presburger formulas,

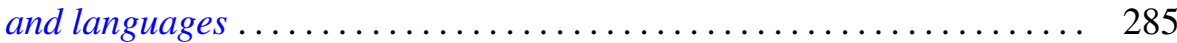

Burrell Washington Helton, Integral equations and product integrals . . . . . . 297

Edgar J. Howard, First and second category Abelian groups with the n-adic topology.........................................

Arthur H. Kruse and Paul William Liebnitz, Jr., An application of a family homotopy extension theorem to ANR spaces.

Albert Marden, I. Richards and Burton Rodin, On the regions bounded by homotopic curves

Willard Miller, Jr., A branching law for the symplectic groups ...

Marc Aristide Rieffel, A characterization of the group algebras of the finite

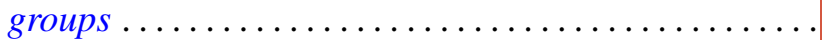

P. P. Saworotnow, On two-sided $H^{*}$-algebras

John Griggs Thompson, Factorizations of p-solvable groups ...

Shih-hsiung Tung, Harnack's inequalities on the classical Cartan

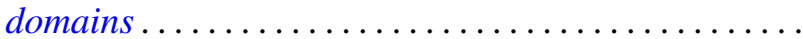

